PlastOx 2007 (2009) 1-21

(C) EDP Sciences, 2009

DOI: $10.1051 /$ ptox/2009002

\title{
Plasticité : rappels de base et aspects microscopiques
}

\author{
B. Viguier
}

CIRIMAT, CNRSIUPSIINPT, ENSIACET, 118 route de Narbonne, 31077 Toulouse Cedex 4, France

\begin{abstract}
Résumé. Dans cet article d'introduction, nous rappelons les définitions et les propriétés générales des états de contrainte et de déformation tels qu'utilisés pour décrire l'état mécanique d'un matériau. Nous introduisons ensuite la notion de dislocation, en présentant dans un premier temps leurs propriétés géométriques puis le champ de déformation qui leur est associé et les propriétés qui en découlent. Nous montrons comment la déformation plastique des cristaux est expliquée par le mouvement des dislocations, particulièrement par le glissement. Différents types de modélisation de la déformation sont mentionnés en se référant aux travaux récents de la littérature. Enfin nous abordons la montée des dislocations, via le mouvement de crans et en présentant les liens avec la concentration de lacunes.
\end{abstract}

\section{INTRODUCTION}

Les caractéristiques mécaniques d'une structure rendent compte de la façon dont des efforts extérieurs (des forces) sont reliés à des déplacements. Ces grandeurs extensives sont remplacées au sein des matériaux par les grandeurs locales que sont les contraintes et les déformations qui caractérisent l'état mécanique de la matière en un point donné. Les différentes relations entre les états de contrainte et de déformation définissent les lois de comportement. Dans les différents chapitres de cet ouvrage, vous verrez comment ces lois de comportement s'écrivent à l'échelle d'une structure ou d'un élément de structure ainsi que la façon dont on écrit ces lois de comportements en tenant compte du caractère localisé et anisotrope de la déformation plastique qui implique les systèmes de glissement. Le passage de la plasticité du monocristal à celle du polycristal sera aussi abordé. De façon à comprendre au mieux les différents mécanismes mis en jeu lors de la déformation plastique des métaux par le mouvement des dislocations il est souvent utile de faire appel à des notions fines nécessitant des techniques de simulation à l'échelle atomique ou à l'échelle mésoscopique (échelle du micromètre : celle des interactions entre segments de dislocations ou entre dislocations et précipités). De nombreuses contributions dans cet ouvrage permettent aussi de mettre en évidence la modification du comportement mécanique des matériaux du fait de leur réactivité avec le milieu environnant. Le lecteur familier avec la mécanique et la théorie des dislocations pourra allègrement passer le présent chapitre pour aborder directement chacun des aspects décrits dans les chapitres suivants. Cependant il nous a paru utile de rappeler ici certaines notions de base de la description des états mécaniques et de la théorie des dislocations qui permettront aux personnes moins coutumières de ce formalisme d'être plus à l'aise pour aborder les chapitres suivants. Les plus curieux trouveront des informations plus complètes dans des ouvrages spécifiques [1-6]. Ce chapitre est construit autour de quatre paragraphes principaux. Dans le paragraphe 2, nous commençons par définir les grandeurs contrainte et déformation, leur expression sous forme tensorielle et nous rappelons quelques propriétés essentielles de ces tenseurs. Nous présentons ensuite au paragraphe 3 les dislocations comme des défauts géométriques au sein du cristal, auxquels est associé un champ de déformation élastique. Nous examinons les propriétés des dislocations selon ces deux aspects (défauts géométriques et champ de déformation). Le rôle du déplacement des dislocations dans la plasticité des cristaux est décrit dans le paragraphe 4 avec une attention particulière sur le glissement. 


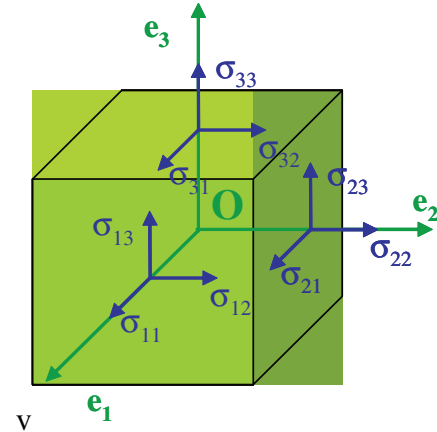

(a)

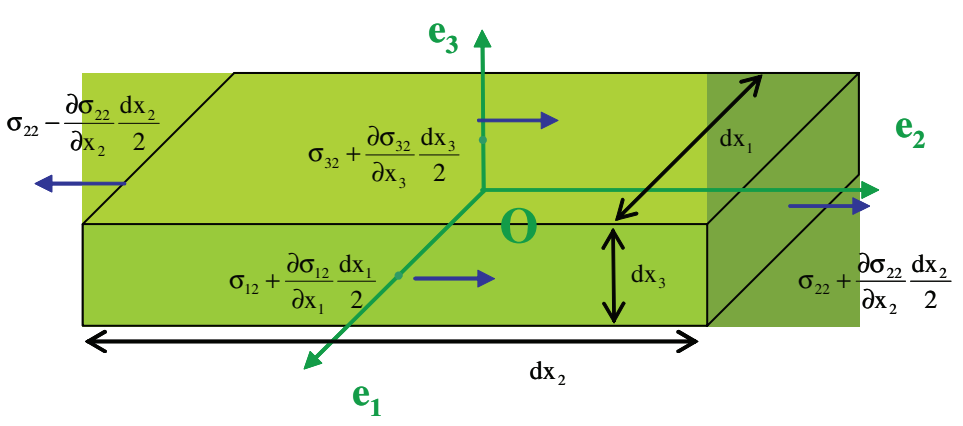

(b)

Figure 1. Définition des composantes du tenseur des contraintes autour d'un pont $\mathrm{O}$ (a) et variation des composantes sur un volume $\left(\mathrm{dx}_{1}, \mathrm{dx}_{2}, \mathrm{dx}_{3}\right)(\mathbf{b})$.

\section{CONTRAINTE ET DÉFORMATION}

\subsection{Définition des tenseurs}

\subsubsection{Contraintes}

Pour décrire l'état de contrainte en un point d'un solide considérons, autour de ce point, un cube infinitésimal qui s'appuie sur les trois directions $\mathbf{e}_{\mathbf{j}}$ d'un trièdre orthonormé. Considérons les forces exercées par le reste du solide sur les faces de ce cube, pour éviter le déplacement du cube, deux faces opposées doivent être soumises à deux forces égales et opposées. Il suffit donc de considérer seulement trois faces du cube. La force $\mathbf{F}$ qui s'exerce sur une face du cube de surface $S_{i}$ et de normale $\mathbf{e}_{\mathbf{i}}$ peut se projeter selon les trois directions $\mathbf{e}_{\mathbf{j}}$, ce qui correspond aux composantes $F_{\mathbf{j}}$. On en déduit une contrainte $: \sigma_{\mathrm{ij}}=\mathrm{F}_{\mathrm{j}} / \mathrm{s}_{\mathrm{i}}$. Définir un état de contrainte général en un point donné nécessite donc de connaître les composantes de la force appliquée sur trois faces perpendiculaires du cube, ce qui correspond aux neuf composantes qui définissent un tenseur de rang deux, figure 1(a). Ce tenseur des contraintes est noté avec deux flèches ou souligné deux fois (pour indiquer son rang). Plus simplement dans de nombreux ouvrages on souligne une fois, ou on note en gras toutes les grandeurs tensorielles, le rang de chaque tenseur étant connu implicitement. On peut aussi écrire le tenseur en donnant l'ensemble de ses composantes:

$$
\overrightarrow{\vec{\sigma}}=\underline{\underline{\sigma}}=\underline{\sigma}=\boldsymbol{\sigma}=\left(\begin{array}{lll}
\sigma_{11} & \sigma_{12} & \sigma_{13} \\
\sigma_{21} & \sigma_{22} & \sigma_{23} \\
\sigma_{31} & \sigma_{32} & \sigma_{33}
\end{array}\right)
$$

Dans cette représentation, les composantes de la diagonale $\sigma_{\text {ii }}$ sont des contraintes normales, et par convention on note positivement les contraintes de traction. Les composantes hors diagonale $\left(\sigma_{\mathrm{ij}}\right.$ avec $\mathrm{i} \neq \mathrm{j}$ ) sont des contraintes de cisaillement ou cissions. Notons que pour assurer l'absence de couple sur le cube infinitésimal de la figure 1 les contraintes de cisaillement qui s'exercent sur deux faces voisines doivent se compenser $\left(\sigma_{\mathrm{ij}}=\sigma_{\mathrm{ji}}\right)$; autrement dit le tenseur des contraintes est symétrique.

Considérons à présent les variations ded contraintes dans un parallélogramme de dimensions $\left(\mathrm{dx}_{1}, \mathrm{dx}_{2}, \mathrm{dx}_{3}\right)$ centré en $\mathrm{O}$, figure $1(\mathrm{~b})$. A une distance $\mathrm{dx}_{i} / 2$ de $\mathrm{O}$ la composante ij du tenseur des contraintes vaut : $\sigma_{\mathrm{ij}}+\frac{\partial \sigma_{\mathrm{ij}}}{\partial \mathrm{x}_{\mathrm{i}}} \frac{\mathrm{d} \mathrm{g}_{i}}{2}$. En l'absence de force de volume au sein du parallélogramme, on doit avoir un équilibre mécanique, c'est-à-dire que la somme des forces appliquées sur les faces du parallélogramme est nulle. Pour les composantes de la force le long de l'axe $\mathbf{e}_{1}$, cet équilibre 


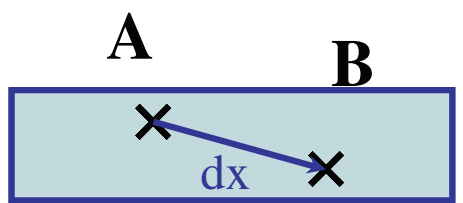

(a)

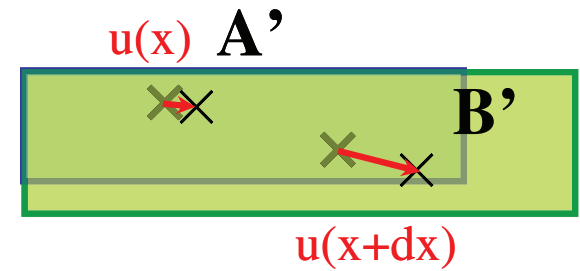

(b)

Figure 2. Schéma permettant de définir le tenseur des déformations. Les deux points A et B du solide non déformés (a) correspondent aux points A' et B' dans le solide déformé (b).

s'écrit : $\frac{\partial \sigma_{11}}{\partial \mathrm{x}_{1}}+\frac{\partial \sigma_{21}}{\partial \mathrm{x}_{2}}+\frac{\partial \sigma_{31}}{\partial \mathrm{x}_{3}}=0$. Appliqué aux trois directions $\mathbf{e}_{\mathbf{i}}$, cette relation peut s'écrire :

$$
\operatorname{div} \underline{\sigma}=0
$$

L'equation (2) est une écriture locale de l'équilibre mécanique des forces au sein du solide. En présence de forces de volume $\mathbf{F}_{\mathbf{j}}$ (forces gravitationnelle, magnétique ou électrostatique) l'élément de volume peut entrer en mouvement. Les équations d'équilibre deviennent alors $: \operatorname{div} \underline{\sigma}+\mathrm{F}_{\mathrm{j}}=\rho \frac{\mathrm{d}^{2} \mathrm{u}_{\mathrm{j}}}{\mathrm{dt}^{2}}$ (où $\rho$ est la densité du solide et $\mathbf{u}$ le vecteur déplacement).

\subsubsection{Déformations}

Pour établir la forme du tenseur des déformations $\underline{\varepsilon}$, considérons $\mathrm{d}$ eux points $\mathrm{A}$ et $\mathrm{B}$ distants de $\mathrm{dx}$ au sein d'un solide : figure 2(a). Dans le solide déformé, figure 2(b), k les points correspondants $\mathrm{A}^{\prime}$ et $\mathrm{B}^{\prime}$ se sont déplacés des vecteur $\mathrm{u}(\mathrm{x})$ et $\mathrm{u}(\mathrm{x}+\mathrm{dx})$ respectivement. La déformation relative e correspond aux déplacements relatifs des points du solide les uns par rapport aux autres :

$$
\mathrm{e}=\frac{\Delta \mathrm{L}}{\mathrm{L}}=\frac{\mathrm{A}^{\prime} \mathrm{B}^{\prime}-\mathrm{AB}}{\mathrm{AB}}=\frac{\mathrm{u}(\mathrm{x}+\mathrm{dx})-\mathrm{u}(\mathrm{x})}{\mathrm{dx}}, \quad \text { soit }: \underline{\mathrm{e}}=\vec{\nabla} \overrightarrow{\mathrm{u}} .
$$

Cependant ce champ de déformation contient une partie antisymétrique $\left(\omega_{\mathrm{ij}}=\frac{1}{2}\left(\frac{\partial \mathrm{u}_{\mathrm{i}}}{\partial \mathrm{x}_{\mathrm{j}}}-\frac{\partial \mathrm{u}_{\mathrm{j}}}{\partial \mathrm{x}_{\mathrm{i}}}\right)\right)$ qui correspond à une rotation rigide du solide. Le tenseur des déformations à proprement parler correspond à la seule partie symétrique de la dérivée des déplacements : $\left(\varepsilon_{\mathrm{ij}}=\frac{1}{2}\left(\frac{\partial \mathrm{u}_{\mathrm{i}}}{\partial \mathrm{x}_{\mathrm{j}}}+\frac{\partial \mathrm{u}_{\mathrm{j}}}{\partial \mathrm{x}_{\mathrm{i}}}\right)\right)$.

De façon analogue au tenseur des contraintes, nous pouvons écrire le tenseur des déformations :

$$
\overrightarrow{\vec{\varepsilon}}=\underline{\varepsilon}=\underline{\varepsilon}=\boldsymbol{\varepsilon}=\left(\begin{array}{lll}
\varepsilon_{11} & \varepsilon_{12} & \varepsilon_{13} \\
\varepsilon_{21} & \varepsilon_{22} & \varepsilon_{23} \\
\varepsilon_{31} & \varepsilon_{32} & \varepsilon_{33}
\end{array}\right)
$$

Les composantes de la diagonale $\varepsilon_{\mathrm{ii}}$ sont des déformations normales ou dilatations, comptées positivement en traction (expansion). Les composantes hors diagonale $\left(\varepsilon_{\mathrm{ij}}\right.$ avec $\left.\mathrm{i} \neq \mathrm{j}\right)$ sont des déformations de cisaillement.

\subsection{Propriétés des tenseurs symétriques de rang deux}

Les tenseurs des contraintes et des déformations sont des tenseurs symétriques de rang 2 , à composantes réelles. Leurs composantes sont donc représentées par une matrice $(3 \times 3)$ symétrique à coefficients réels. Une telle matrice est toujours diagonalisable et admet des invariants, ces propriétés sont décrites ci-dessous pour les composantes du tenseur des contraintes. Cette démarche est évidemment transposable au tenseur des déformations. 
Pour un état de contrainte donné, le tenseur $\underline{\sigma}$ est unique, mais ses composantes $\sigma_{\mathrm{ij}}$ dépendent du repère $\left\{\mathbf{e}_{i}\right\}$ choisi pour les exprimer. Lorsqu'on diagonalise la matrice représentant les coordonnées du tenseur, on exprime ce tenseur dans un repère particulier appelé le repère principal. Dans ce repère principal seules les contraintes normales sont non nulles, ce sont les contraintes principales, on les note usuellement $\sigma_{\mathrm{I}}, \sigma_{\mathrm{II}}$ et $\sigma_{\mathrm{III}}$ avec $\sigma_{\mathrm{I}}>\sigma_{\mathrm{II}}>\sigma_{\mathrm{III}}$.

$$
\left(\begin{array}{lll}
\sigma_{11} & \sigma_{12} & \sigma_{13} \\
\sigma_{21} & \sigma_{22} & \sigma_{23} \\
\sigma_{31} & \sigma_{32} & \sigma_{33}
\end{array}\right)_{\substack{\text { repère } \\
\text { quelconque }}} \rightarrow\left(\begin{array}{ccc}
\sigma_{\mathrm{I}} & 0 & 0 \\
0 & \sigma_{\mathrm{II}} & 0 \\
0 & 0 & \sigma_{\mathrm{III}}
\end{array}\right)_{\substack{\text { repère } \\
\text { principal }}}
$$

Le premier invariant du tenseur des contraintes correspond à la trace de la matrice de ses composantes. Cette valeur vaut trois fois la pression hydrostatique :

$$
\mathrm{I}_{1}=\operatorname{Tr}\{\sigma\}=\sigma_{11}+\sigma_{22}+\sigma_{33}=3 \mathrm{P}
$$

Pour le tenseur des déformations ce premier invariant correspond à la somme des dilatations. Dans le cas des faibles déformations (ce qui est le cas des déformations élastiques dans les matériaux cristallins) cette somme correspond à une variation relative de volume :

$$
\mathrm{I}_{1}=\operatorname{Tr}\{\varepsilon\}=\varepsilon_{11}+\varepsilon_{22}+\varepsilon_{33} \approx \Delta \mathrm{V} / \mathrm{V}
$$

Le second invariant du tenseur des contrainte $\left(\mathrm{I}_{2}=\sigma_{\mathrm{ik}} \sigma_{\mathrm{ki}}-\sigma_{\mathrm{ii}} \sigma_{\mathrm{kk}}\right)$ est utilisé pour calculer les valeurs des contraintes principales. La contrainte équivalente de Von Mises correspond au second invariant de la partie déviatorique du tenseur des contraintes (paragraphe ci-dessous).

$$
\sigma_{\mathrm{VM}}=\frac{1}{\sqrt{2}} \sqrt{\left(\left(\sigma_{\mathrm{I}}-\sigma_{\mathrm{II}}\right)^{2}+\left(\sigma_{\mathrm{II}}-\sigma_{\mathrm{III}}\right)^{2}+\left(\sigma_{\mathrm{III}}-\sigma_{\mathrm{I}}\right)^{2}\right)}
$$

Un état de contrainte quel qu'il soit peut toujours être séparé en deux composantes selon la relation suivante : $\underline{\sigma}=\operatorname{Tr}\{\sigma\} \cdot \underline{\mathrm{I}}+\{\underline{\sigma}-\operatorname{Tr}\{\sigma\} \cdot \underline{\mathrm{I}}\}$, où $\underline{\mathrm{I}}$ représente le tenseur unité. Le premier terme de la décomposition $(\operatorname{Tr}\{\sigma\} \cdot \underline{\mathrm{I}})$ correspond ainsi à la pression hydrostatique seule, il s'agit donc d'un terme purement isotrope souvent qualifié de partie «sphérique» du tenseur des contraintes. Le second terme possède une trace nulle, c'est-à-dire que la somme des contraintes normales est nulle, l'état de contrainte est purement de cisaillement ; on appelle ce terme la partie «déviatorique » (ou déviateur) du tenseur des contraintes. On peut représenter cette décomposition selon l'expression :

$$
\left(\begin{array}{lll}
\sigma_{11} & \sigma_{12} & \sigma_{13} \\
\sigma_{21} & \sigma_{22} & \sigma_{23} \\
\sigma_{31} & \sigma_{32} & \sigma_{33}
\end{array}\right)=\left(\begin{array}{lll}
\mathrm{P} & 0 & 0 \\
0 & \mathrm{P} & 0 \\
0 & 0 & \mathrm{P}
\end{array}\right)+\left(\begin{array}{lll}
\sigma_{11}^{\prime} & \tau_{12} & \tau_{13} \\
\tau_{21} & \sigma_{22}^{\prime} & \tau_{23} \\
\tau_{31} & \tau_{32} & \sigma_{33}^{\prime}
\end{array}\right)
$$

(où l'on a réécrit les contraintes pour la partie déviatorique : $\sigma_{i i}^{\prime}=\sigma_{i i}-P$ pour les contraintes normales et $\tau_{i j}=\sigma_{i j}$ pour les contraintes de cisaillement ; on vérifie bien que la trace de cette matrice est nulle). Il est très important de remarquer que ces deux parties de la contrainte peuvent être considérées de façons indépendantes.

Une décomposition analogue peut être réalisée pour le tenseur des déformations. La partie sphèrique correspond alors à un changement de volume : dilatation - ou contraction - isotrope d'un solide sans changement de forme. La partie déviatorique correspondant à un changement de forme à volume constant. Nous pouvons écrire (avec la même écriture que ci-dessus pour la partie déviatorique) :

$$
\left(\begin{array}{lll}
\varepsilon_{11} & \varepsilon_{12} & \varepsilon_{13} \\
\varepsilon_{21} & \varepsilon_{22} & \varepsilon_{23} \\
\varepsilon_{31} & \varepsilon_{32} & \varepsilon_{33}
\end{array}\right)=\frac{1}{3}\left(\begin{array}{ccc}
\Delta \mathrm{V} / \mathrm{v} & 0 & 0 \\
0 & \Delta \mathrm{V} / \mathrm{v} & 0 \\
0 & 0 & \Delta \mathrm{V} / \mathrm{v}
\end{array}\right)+\left(\begin{array}{lll}
\varepsilon_{11}^{\prime} & \gamma_{12} & \gamma_{13} \\
\gamma_{21} & \varepsilon_{22}^{\prime} & \gamma_{23} \\
\gamma_{31} & \gamma_{32} & \varepsilon_{33}^{\prime}
\end{array}\right)
$$

Cette décomposition justifie la nécessité de deux modules indépendants pour l'élasticité isotrope : le module de compressibilité (B) qui relie les parties sphériques et le module de cisaillement $(\mu)$ qui relie les parties déviatoriques. Cette décomposition est aussi utile en plasticité puisque seule la 
partie déviatorique des tenseurs concerne le glissement des dislocations alors que les parties sphériques interviennent dans les phénomènes de diffusion et la montée des dislocations.

\section{DISLOCATIONS DANS LE RÉSEAU : DÉFINITION ET PROPRIÉTÉS}

Dans son sens le plus général, la déformation plastique des matériaux englobe toutes les déformations à caractère permanent (par opposition à la déformation élastique qui est recouvrable). Dans la très vaste majorité des cas, la déformation plastique dans les matériaux cristallins est associée au mouvement des dislocations (citons comme mécanismes alternatifs le maclage, la plasticité de transformation de phase ...). La déformation plastique associée au mouvement des dislocations se caractérise par une localisation de la déformation macroscopique entre plans cristallins qui est révélée par la présence de traces de glissement à la surface des matériaux déformés. Un calcul simpliste basé sur l'extension des relations de l'élasticité cristalline indique que pour réaliser un tel glissement plan sur plan de façon rigide, il faudrait exercer une contrainte de cisaillement théorique :

$$
\tau_{\mathrm{th}}=\frac{\mu b}{2 \pi d} \approx \mu / 5,
$$

où $\mathrm{b}$ représente la distance interatomique et $\mathrm{d}$ la distance interéticulaire [2]. Cette valeur est bien plus élevée que la limite d'élasticité expérimentale des métaux (qui vaut de l'ordre de $\mu / 10000$ à $\mu / 1000$ ). Ce désaccord indique qu'il existe un mécanisme qui facilite le glissement plan sur plan. Ce mécanisme correspond au mouvement des dislocations qui permet une localisation de la déformation au sein du plan cristallographique.

\subsection{Caractéristiques géométriques}

La notion de dislocations a été introduite en mécanique des milieux continus au début du $20^{\text {me }}$ siècle, il s'agit d'une ligne de singularité d'un champ de déformation élastique (et donc de contrainte) [7]. Un tel défaut, que l'on appelle dislocation de Voltera, peut être créé au sein d'un matériau homogène en effectuant une coupure limitée par une courbe $L$ au sein du matériau. Les deux surfaces $S_{1}$ et $S_{2}$ qui limitent la coupure sont déplacées l'une par rapport à l'autre de façon rigide ${ }^{1}$, on ajoute ou enlève éventuellement de la matière où cela est nécessaire pour éviter tout vide ou recouvrement. Des propriétés générales très importantes des dislocations découlent naturellement de cette façon de les créer : i) la dislocation est un défaut linéique caractérisé par deux vecteurs : le vecteur de ligne (noté $\xi$ et tangent en tout point à la ligne L) et le vecteur de déplacement (que nous noterons b : vecteur de Burgers) et ii) le vecteur de déplacement est constant le long de la ligne, ce qui implique qu'une ligne de dislocation ne peut se terminer au sein du matériau mais doit déboucher sur un défaut (surface, joint de grain, précipité, autre dislocation - dans ce dernier cas, on retrouve une « loi des nœuds » analogue à celle des courants électriques dans un circuit). On qualifie une dislocation de coin lorsque $\boldsymbol{\xi}$ et $\mathbf{b}$ sont perpendiculaires, de vis lorsqu'ils sont parallèles, figure 3 ; dans les autres cas, on parle de dislocation mixte de caractère $\theta$, où $\theta$ est l'angle entre $\boldsymbol{\xi}$ et $\mathbf{b}$.

Autour des années 1930-1940, cette notion de dislocation a été transposée aux matériaux cristallins de façon à expliquer leur comportement plastique. Le caractère cristallin implique une seule particularité : les liaisons atomiques ne sont perturbées que localement autour de la ligne de dislocation que si le vecteur de déplacement $\mathbf{b}$ - ou vecteur de Burgers - est un vecteur du réseau. On parle alors de dislocation parfaite. Dans le cas contraire, la dislocation est dite partielle et elle s'accompagne d'un défaut surfacique de nature géométrique (défaut d'empilement) ou chimique (paroi d'antiphase). On mesure la quantité de dislocations au sein d'un cristal par leur densité $\rho$, qui correspond au le rapport

\footnotetext{
${ }^{1}$ Plus strictement, le défaut créé est une dislocation si le déplacement imposé est une translation ; s'il s'agit d'une rotation on a alors affaire à une disinclinaison ou «disclinaison » que l'on rencontre fréquemment dans certains matériaux polymères [8].
} 


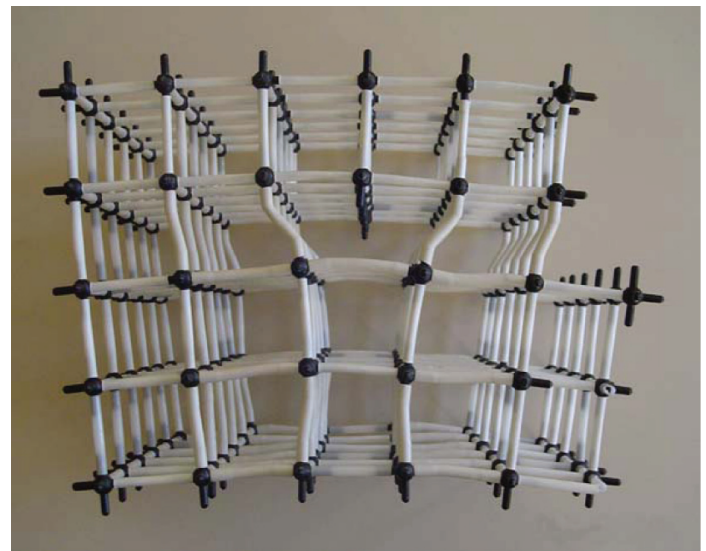

(a)

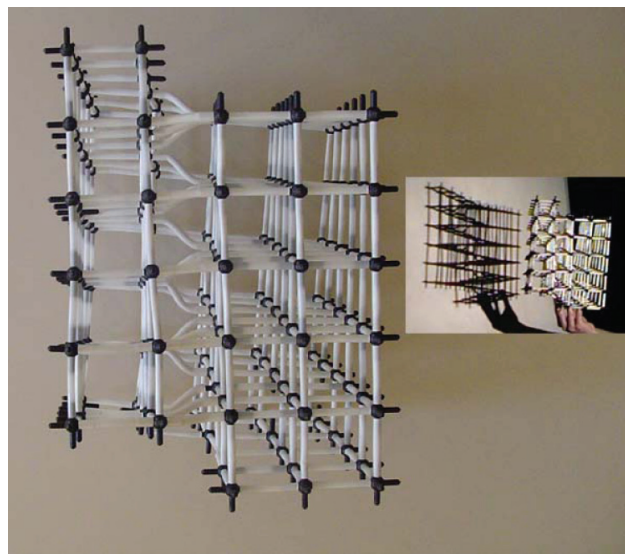

(b)

Figure 3. Exemple de dislocations coin (a) et vis (b) dans un modèle de cristal (l'ombre projetée du modèle de la dislocation vis montre bien « la vis» dessinée par les plans atomiques).

entre la longueur totale de ligne de dislocations $\mathrm{L}$ et le volume du cristal V :

$$
\rho=\mathrm{L} / \mathrm{V}
$$

La notion de réseau de Franck permet de donner une idée plus précise de ce que représente la densité de dislocations : si on considère au sein d'un cristal de volume $\mathrm{V}$ des dislocations rectilignes, parallèles et arrangées selon un réseau carré, la maille de réseau $\mathrm{d}_{\text {Franck }}$ (distance entre dislocations) vaut alors :

$$
\mathrm{d}_{\text {Franck }}=1 / \sqrt{\rho} .
$$

Ainsi pour un métal bien recuit (qui contient une très faible quantité de dislocations) on a $\rho \approx 10^{10} \mathrm{~m}^{-2}$ soit $\mathrm{d}_{\text {Franck }} \approx 10 \mu \mathrm{m}$; alors que dans le même métal fortement écroui $\rho \approx 10^{14} \mathrm{~m}^{-2}$ soit $\mathrm{d}_{\text {Franck }} \approx$ $100 \mathrm{~nm}$. On peut remarquer aussi que la distance moyenne entre dislocations étant nécessairement grande devant la distance interatomique il existe une borne supérieure à la densité de dislocations $\left(\rho_{\operatorname{Max}} \approx 10^{18} \mathrm{~m}^{-2}\right)$.

\subsection{Bases de la théorie élastique des dislocations}

Nous avons décrit les dislocations comme des lignes de défaut correspondant à des liaisons perturbées au sein du cristal, tel qu'illustré sur la figure 3. Cette vision permet de visualiser le déplacement (vecteur de Burgers) qui leur est associé aux dislocations. Cependant les propriétés des dislocations dépendent très largement du champ de déformation élastique qui entoure ces lignes de défaut, en particulier parce qu'il fixe les interactions à distance avec tous les éléments qui présentent un champ de contrainte. Nous allons présenter ces propriétés dans le cadre de l'élasticité isotrope, en effet la prise en compte de l'anisotropie cristalline des coefficients d'élasticité alourdit considérablement les calculs et nécessite en général une solution numérique (voir par exemple un programme de calcul d'énergie et de tension de ligne des dislocations permettant de déterminer les formes d'équilibre des boucles [9]).

\subsubsection{Champ de déformation et énergie élastique}

Une première approximation du champ de contrainte associé aux dislocations est obtenue très simplement dans le cas d'une dislocation vis, rectiligne et supposée infinie. En utilisant la géométrie et les repères de la figure 4, le champ de déplacement induit par la dislocation vis s'exprime simplement 


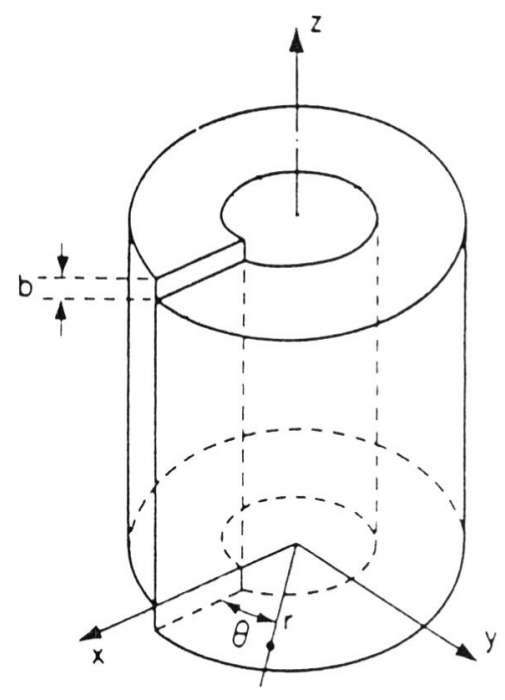

(a)

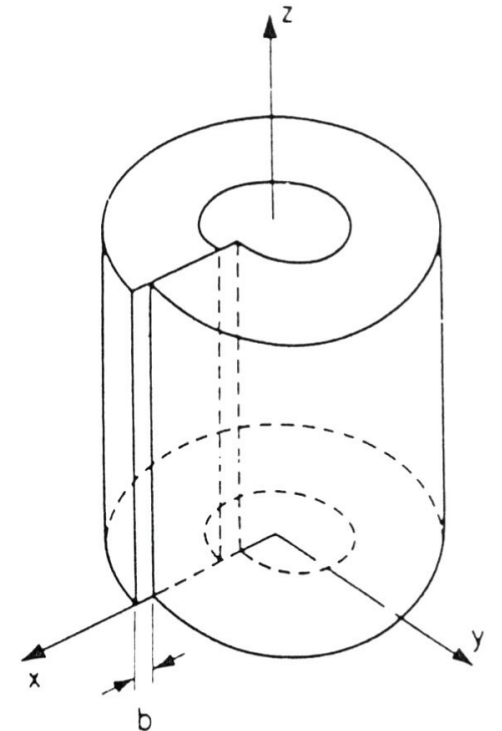

(b)

Figure 4. Géométrie et repères utilisés pour le calcul des déformations et des contraintes associées aux dislocations vis (a) et coin (b).

en coordonnées cylindriques : $\mathbf{u}=\mathrm{u}_{\theta} \mathbf{e}_{\mathbf{z}}=\mathrm{b} \frac{\theta}{2 \pi} \mathbf{e}_{\mathbf{z}}$. La seule composante non nulle des tenseurs des déformations et des contraintes est donc :

$$
\varepsilon_{\mathrm{z} \theta}^{\mathrm{v}}=\varepsilon_{\theta \mathrm{z}}^{\mathrm{v}}=\frac{\mathrm{b}}{4 \pi \mathrm{r}} \quad \text { et } \quad \sigma_{\mathrm{z} \theta}^{\mathrm{v}}=\sigma_{\theta \mathrm{z}}^{\mathrm{v}}=\frac{\mu \mathrm{b}}{2 \pi \mathrm{r}}
$$

En coordonnées cartésiennes les composantes non nulles sont, pour le tenseur des contraintes :

$$
\sigma_{\mathrm{xz}}^{\mathrm{v}}=\sigma_{\mathrm{zx}}^{\mathrm{v}}=-\frac{\mu \mathrm{b}}{2 \pi} \frac{\mathrm{y}}{\left(\mathrm{x}^{2}+\mathrm{y}^{2}\right)} \quad \text { et } \quad \sigma_{\mathrm{yz}}^{\mathrm{v}}=\sigma_{\mathrm{zy}}^{\mathrm{v}}=+\frac{\mu \mathrm{b}}{2 \pi} \frac{\mathrm{x}}{\left(\mathrm{x}^{2}+\mathrm{y}^{2}\right)} .
$$

Il apparaît donc que le champ de contraintes d'une dislocation vis correspond à des cissions pures, c'est-à-dire qu'une dislocation vis ne sera pas sensible à des variation de pression hydrostatique.

Le champ de déformation associé à une dislocation coin est bien plus complexe que celui d'une vis. On ne peut pas énoncer intuitivement le champ des déplacements, mais les conditions d'équilibre de la mécanique associées aux conditions aux limites correspondant à la dislocation coin permettent de déduire un champ de contrainte. En coordonnées cartésiennes, les seules composantes non nulles sont (avec $v$ coefficient de Poisson) :

$$
\begin{gathered}
\sigma_{\mathrm{xx}}^{\mathrm{C}}=-\frac{\mu \mathrm{b}}{2 \pi(1-v)} \frac{\mathrm{y}\left(3 \mathrm{x}^{2}+\mathrm{y}^{2}\right)}{\left(\mathrm{x}^{2}+\mathrm{y}^{2}\right)^{2}} ; \quad \sigma_{\mathrm{yy}}^{\mathrm{C}}=+\frac{\mu \mathrm{b}}{2 \pi(1-v)} \frac{\mathrm{y}\left(\mathrm{x}^{2}-\mathrm{y}^{2}\right)}{\left(\mathrm{x}^{2}+\mathrm{y}^{2}\right)^{2}} ; \quad \sigma_{\mathrm{zz}}^{\mathrm{C}}=v\left(\sigma_{\mathrm{xx}}^{\mathrm{C}}+\sigma_{\mathrm{yy}}^{\mathrm{C}}\right) \\
\text { et } \sigma_{\mathrm{xy}}^{\mathrm{C}}=\sigma_{\mathrm{yx}}^{\mathrm{C}}=+\frac{\mu \mathrm{b}}{2 \pi(1-v)} \frac{\mathrm{x}\left(\mathrm{x}^{2}-\mathrm{y}^{2}\right)}{\left(\mathrm{x}^{2}+\mathrm{y}^{2}\right)^{2}} .
\end{gathered}
$$

On note tout d'abord que le champ de contrainte associé à la dislocation coin contient des termes de contrainte normale. Il existe donc un champ de pression hydrostatique associé à une dislocation coin 
(ce terme est important pour les interactions des dislocations coins avec les défauts ponctuels - solutés ou lacunes) :

$$
\mathrm{P}^{\mathrm{C}}=-\frac{\mu \mathrm{b}(1+v)}{3 \pi(1-v)} \frac{\mathrm{y}}{\left(\mathrm{x}^{2}+\mathrm{y}^{2}\right)} .
$$

C'est une contrainte de compression au dessus du plan qui contient le vecteur de Burgers et de traction en dessous. On vérifie aisément que ces contraintes se compensent : la pression hydrostatique intégrée sur un cylindre de rayon $r$ centré sur la ligne de dislocation est nulle. On vérifie aussi que la présence d'une dislocation dans un cristal n'induit globalement pas de variation de contrainte normale, c'està-dire pas de variation de volume (il conviendrait toutefois de tenir compte d'une faible variation de volume due au cœur des dislocations).

En comparant les champs de contrainte des dislocations vis et coin, orientées selon la même direction, on constate que les composantes non nulles pour une dislocation sont nulles pour l'autre. Ces champs sont dits orthogonaux, c'est-à-dire qu'il n'y a pas d'interaction élastique entre une dislocation vis et une dislocation coin parallèles. Par suite, le champ de contrainte $\underline{\boldsymbol{\sigma}}^{\mathrm{M}}$ autour d'une dislocation mixte de caractère $\theta$ s'écrira simplement en additionnant le champ de contrainte de la partie vis $\left(\mathrm{b}_{\mathrm{v}}=\mathrm{b} \cos \theta\right)$ et celui de la partie coin $\left(b_{c}=b \sin \theta\right)$.

L'énergie élastique associée au champ de déformation et de contrainte d'une dislocation s'intègre au sein d'un tube de rayon interne $\mathrm{r}_{\mathrm{C}}$ et de rayon externe $\mathrm{R}$ qui entoure la dislocation on trouve par unité de longueur [1] :

$$
\mathrm{W}=\frac{\mu \mathrm{b}^{2}}{4 \pi \mathrm{K}} \ln \left(\frac{\mathrm{R}}{\mathrm{r}_{\mathrm{c}}}\right) ; \quad \operatorname{avec} \mathrm{K}=\frac{1-v}{1-v \cos ^{2} \theta}
$$

Le facteur $\mathrm{K}$ rend compte de l'influence du caractère de la dislocation. Le rayon $\mathrm{r}_{\mathrm{c}}$ est appelé rayon de cœur de la dislocation : lorsqu'on se rapproche de la ligne de dislocation, les liaisons interatomiques sont perturbées et les lois de l'élasticité macroscopique ne peuvent plus s'appliquer ( $\mathrm{r}_{\mathrm{c}}$ vaut environ $2 \mathrm{~b}$ ). L'énergie associée à ces perturbations est «l'énergie de cœur des dislocations » qui peut être estimée par des calculs atomistiques. Le rayon de coupure externe $\mathrm{R}$ correspond à la distance d'écrantage du champ de déformation, en particulier par les autres dislocations $\left(R \approx \mathrm{d}_{\text {Franck }}\right)$. Les valeurs approchées de $\mathrm{r}_{\mathrm{c}}$ et $\mathrm{R}$ permettent ainsi de donner une bonne estimation de l'énergie totale par unité de longueur, on retient souvent l'expression simplifiée :

$$
\mathrm{W} \approx \frac{\mu \mathrm{b}^{2}}{2}
$$

Notons l'ordre de grandeur de cette énergie de ligne qui vaut environ $5 \mathrm{eV}$ pour une longueur de b, cette énergie considérable justifie que les dislocations, bien que toujours présentes dans les cristaux, sont toujours des défauts hors équilibre thermodynamique (le surcroît d'entropie qu'elles créent est minime par rapport à cette énergie). La variation en $\mathrm{b}^{2} \mathrm{de} \mathrm{W}$ impose que les vecteurs de Burgers que l'on rencontre correspondent dans chaque structure aux vecteurs de translation les plus courts du réseau.

\subsubsection{Interactions élastiques à distance}

Une dislocation correspond donc à un champ de contrainte localisé. Déplacer ce champ de $\delta \mathbf{r}$ sous l'effet d'une contrainte extérieure $\underline{\sigma}$ nécessite d'effectuer un travail $\delta \mathrm{W}$. Il est souvent beaucoup plus simple de représenter cette variation d'énergie par une force thermodynamique virtuelle : $\mathbf{F}=\delta \mathrm{W} / \delta \mathbf{r}$. Cette force virtuelle est connue sous le nom de force de Peach - Kölher, elle s'exprime par unité de longueur de ligne de la dislocation :

$$
\mathbf{F}=(\mathbf{b} \cdot \underline{\boldsymbol{\sigma}}) \times \boldsymbol{\xi}
$$

Cette force est en tout point perpendiculaire à la ligne de la dislocation. Décomposons la contrainte appliquée en ses deux composantes sphérique et déviatorique. On voit que la partie sphérique (pression 
hydrostatique $\mathrm{P}$ ) implique une force de montée pure par unité de longueur de la dislocation : $\mathrm{F}_{\mathrm{M}}=$ $\mathrm{Pb}$ - cette force est donc nulle pour une dislocation vis. La partie déviatorique implique une force de glissement dans le plan de glissement de la dislocation (les géométries du glissement et de la montée sont rappelées au paragraphe 4 ci-dessous). Si $\tau$ est la cission projetée dans le plan de glissement, perpendiculairement à la ligne on a une force de glissement par unité de longueur de ligne de la dislocation : $\mathrm{F}_{\mathrm{G}}=\tau \mathrm{b}$. La force de Peach - Kölher permet d'exprimer l'action d'une contrainte extérieure sur une dislocation mais aussi de tout élément au sein du matériau qui possède un champ de contrainte. On peut ainsi écrire les interactions élastiques entre une dislocation et des précipités, ou entre dislocations. Pour deux dislocations parallèles distantes de R, de vecteurs de Burgers respectifs $\mathbf{b}_{1}$ et $\mathbf{b}_{2}$ et de vecteur de ligne $\boldsymbol{\xi}$, la force d'interaction est essentiellement radiale. Elle s'exprime par unité de longueur:

$$
\mathbf{F}_{\mathrm{R}}=\frac{\mu}{2 \pi \mathrm{R}}\left[\left(\mathbf{b}_{1} \cdot \xi\right)\left(\mathbf{b}_{2} \cdot \xi\right)+\frac{\left(\mathbf{b}_{1} \times \xi\right) \cdot\left(\mathbf{b}_{2} \times \xi\right)}{(1-v)}\right]
$$

Cette force est positive (force de répulsion) lorsque le produit scalaire $\mathbf{b}_{1} \cdot \mathbf{b}_{2}$ est positif.

Nous comprenons à présent facilement que dans la plupart des métaux les dislocations aient tendance à se dissocier pour former des dislocations partielles : la décomposition d'un vecteur de Burgers en deux vecteurs formant un angle aigu est énergétiquement favorable $\left(b^{2}>b_{1}^{2}+b_{2}^{2}\right)$. Comme ces vecteurs de Burgers forment un angle aigu, les partielles exercent l'une sur l'autre une force de répulsion. La largeur de dissociation d est alors fixée par l'énergie du défaut d'empilement créé ( $\gamma_{\mathrm{SF}}$, SF pour stacking fault) en équilibre avec la répulsion élastique des partielles :

$$
\gamma_{\mathrm{SF}}=\frac{\mu}{2 \pi} \frac{1}{\mathrm{~d}}\left[\left(\mathbf{b}_{1} \cdot \xi\right)\left(\mathbf{b}_{2} \cdot \xi\right)+\frac{\left(\mathbf{b}_{1} \times \xi\right) \cdot\left(\mathbf{b}_{2} \times \xi\right)}{(1-v)}\right]
$$

Une faible énergie de défaut d'empilement (c'est le cas des alliages de cuivre ou des aciers austénitiques) implique une dissociation importante et donc un glissement planaire des dislocations. Au contraire, une forte énergie de défaut d'empilement (comme dans l'aluminium ou le nickel) implique une faible dissociation et facilite le glissement dévié et donc tous les phénomènes nécessitant un mouvement non planaire des dislocations : formation de sous joints de grain, restauration.

\subsubsection{Surfaces et couches d'oxyde}

Dans le cadre de cet ouvrage où nous nous intéressons à la réactivité des matériaux, les surfaces et les interfaces jouent un rôle particulier. Les surfaces libres, comme toutes les interfaces, peuvent constituer des discontinuités des champs de contrainte et de déformation et leur rôle dans la plasticité des cristaux est très important [10]. Ainsi, d'un point de vue purement élastique, une dislocation proche d'une surface libre ressent une contrainte qui tend à l'attirer vers la surface. On peut, en première approximation, calculer cette force par la notion de «dislocation image»: comme on le fait en électromagnétisme avec des lignes de courants sous la surface d'un conducteur, on remplace alors la surface libre par une dislocation «image». Un traitement plus rigoureux de ces forces images nécessite des simulations à l'échelle atomique [11]. Pour une dislocation vis, parallèle à la surface et située à une distance d de celle ci, la force d'attraction de la surface vaut $: \mathbf{F}_{\mathrm{S}}=\frac{\mu}{2 \pi} \frac{\mathrm{b}^{2}}{2 \mathrm{~d}}$. Dans le cas d'une interface séparant deux milieux, la force sera attractive si le milieu dans lequel se trouve la dislocation (milieu 1) est plus rigide que l'autre (milieu 2). A contrario, une interface avec un milieu plus rigide que celui dans lequel se trouve la dislocation tend à repousser la dislocation. Dans le cas d'une surface oxydée, les deux phénomènes s'additionnent puisque pour une dislocation près de la surface d'un métal, le vide attire cette dislocation alors que la couche d'oxyde (en général plus rigide que le métal) tend à la repousser. Cette situation nécessite de prendre en compte l'ensemble des interfaces, ce qui correspond à un problème de matériau multicouche [12]. L'importance des deux phénomènes (attraction du vide/répulsion par l'oxyde) dépend très fortement du rapport de rigidité entre le métal et l'oxyde. Ainsi une couche 


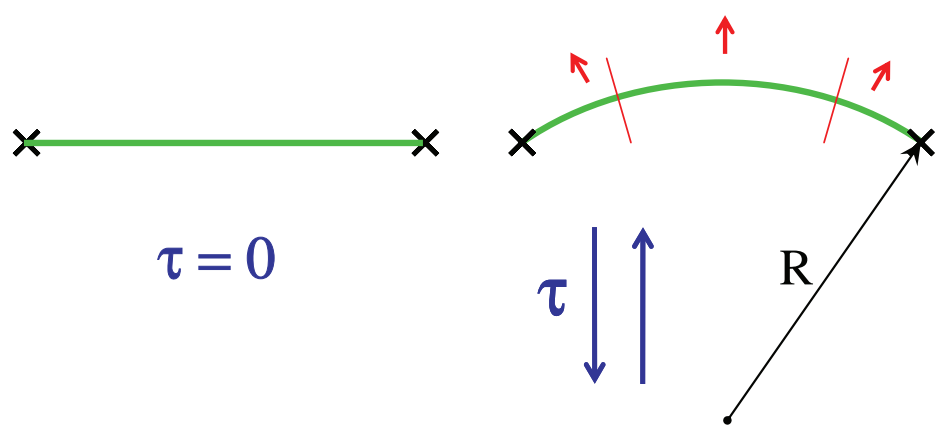

(a) (b)

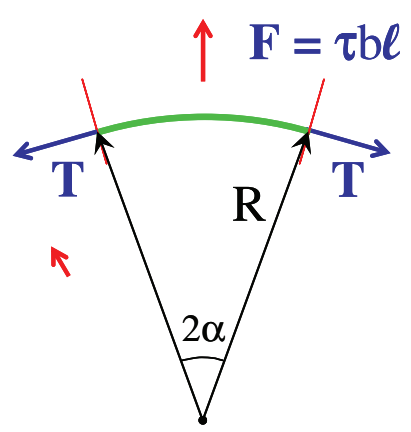

(c)

Figure 5. Le segment de dislocation en (a) se courbe sous l'effet de la contrainte appliquée $\tau$ b). La tension de ligne $\mathrm{T}$ est la force qui maintient un segment de cette dislocation dans sa forme d'équilibre (c), expérimentalement la mesure du rayon de courbure $\mathrm{R}$ permet de déterminer la contrainte locale.

de $5 \mathrm{~nm}$ d'épaisseur d'alumine sur de l'aluminium $\left(\mu_{\text {oxyde }} / \mu_{\text {métal }}=5,67\right)$ suffit à repousser toutes les dislocations vers l'intérieur du cristal ; alors qu'une couche de $100 \mathrm{~nm}$ de $\mathrm{NiO}$ à la surface du nickel $\left(\mu_{\text {oxyde }} / \mu_{\text {métal }}=1,22\right)$ écrante à peine l'effet de la surface libre.

\subsection{Tension de ligne et courbure}

Nous n'avons considéré jusqu'à présent que des dislocations purement rectilignes. En réalité, les dislocations se courbent au sein du cristal, sous l'effet des contraintes et parce que certains points sont ancrés par des obstacles locaux. La tension de ligne d'une dislocation est l'analogue à une dimension de la notion de tension de surface. Cette tension de ligne correspond à la force exercée en un point de la ligne par le reste de la dislocation, c'est une force tangente à la ligne en ce point. Comme la raideur pour un ressort élastique, la tension de ligne résiste à l'allongement et à la courbure de la ligne de dislocation. La tension de ligne dépend aussi du caractère de la dislocation et s'écrit ( $\theta$ est le caractère du segment de dislocations considéré) :

$$
\mathrm{T}(\theta)=\mathrm{W}(\theta)+\frac{\partial^{2} \mathrm{~W}}{\partial \theta^{2}}
$$

Ainsi, dans un milieu isotrope une dislocation vis qui possède une énergie plus faible qu'une dislocation coin sera beaucoup plus stable et les boucles de dislocations ont tendance à s'étirer le long de la direction vis. Dans le cadre de l'élasticité anisotrope, le terme de courbure peut devenir négatif et certaines orientations de ligne de dislocations deviennent instables [9]. Notons que cette notion de tension de ligne, issue d'un raisonnement en termes de force, est en toute rigueur valable seulement pour des dislocations peu courbées et isolées. Dans le cas contraire il vaut mieux passer par des raisonnements basés sur l'énergie des dislocations [13].

Le rayon de courbure est ainsi directement lié à la contrainte que subit la dislocation. Considérons le segment de dislocation de la figure 5 a qui est bloqué à ses extrémités. Lorsque l'on exerce une contrainte de cisaillement $\tau$, la ligne de dislocation est soumise à la force de Peach - Kölher en tout point perpendiculaire à sa ligne et la ligne se courbe (fig. 5b). Si on isole un segment de cette dislocation courbée, on peut calculer l'équilibre mécanique entre cette force de Peach et Kölher intégrée sur le segment de longueur $\ell$ et la tension de ligne de la dislocation : $F_{\mathrm{PK}} \ell=\tau \mathrm{b} \ell=\tau \mathrm{bR} \cdot 2 \alpha=$ $\mathrm{T}_{\text {projeté }}=2 \mathrm{~T} \cdot \sin \alpha($ pour de faibles valeurs de $\alpha)$, en approximant la tension de ligne $\mathrm{T} \approx \mathrm{W} \approx \frac{\mu \mathrm{b}^{2}}{2}$. 


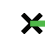

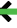

(a)

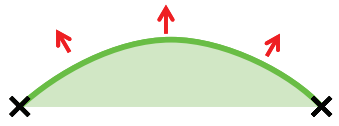

(b)

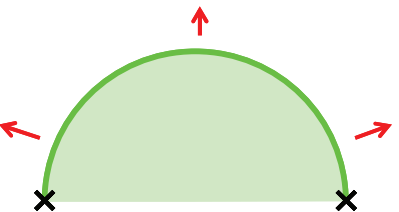

(c)

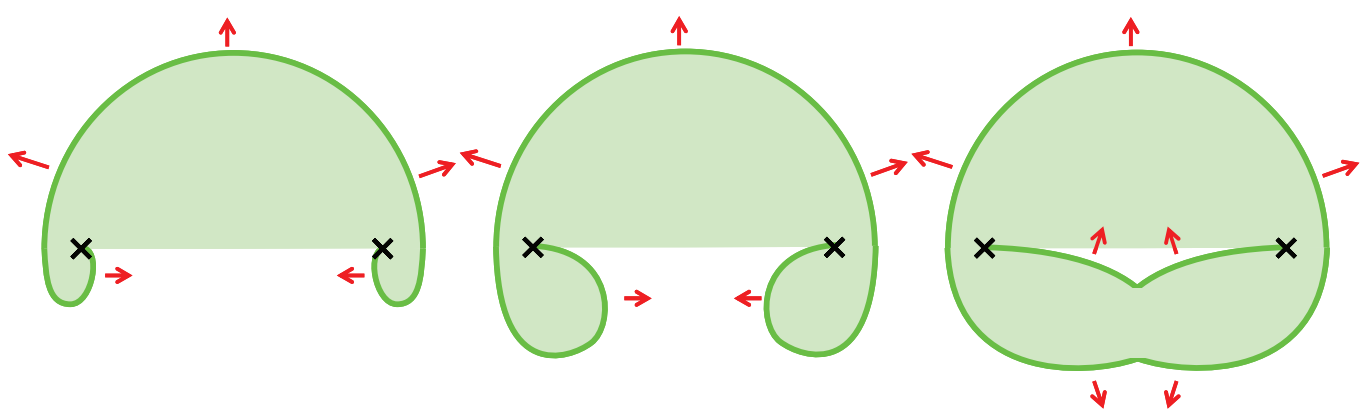

(d)

(e)

(f)

Figure 6. Schéma de fonctionnement du moulin de Frank - Read. Le segment de dislocation ancré en deux points (a) se courbe sous l'effet d'une contrainte appliquée (b) jusqu'à atteindre une position critique (c) pour laquelle le rayon de courbure est minimum. Au-delà les segments situés sous les points d'ancrage s'attirent (d) et (e) jusqu'à s'annihiler pour donner une boucle fermée et un nouveau segment indépendant (f).

On obtient :

$$
\mathrm{R}=\frac{\mu \mathrm{b}}{2 \tau}
$$

La courbure des dislocations est utilisée pour mesurer la contrainte locale au sein d'un matériau. Cette technique est ainsi largement utilisée pour dépouiller les essais de déformation plastique réalisés in situ dans le microscope électronique en transmission [14].

Ce calcul permet de déterminer la contrainte seuil de fonctionnement du moulin de Frank - Read qui est illustré sur la figure 6. Un segment de dislocation, coincé par deux points d'ancrage distants de D (fig. 6a) se courbe sous l'effet de la contrainte appliquée (figs. 6(b) et (c)). Le point critique correspondant à la courbure maximale du segment de dislocation correspond à la configuration en demi cercle de la fig. 6(c). Au-delà, la courbure diminue, les segments de dislocations en vis-à-vis s'attirent élastiquement et finalement s'annihilent (figs. 6(d) et (e)). Ce mécanisme est à la base de la plupart des mécanismes de multiplication des dislocations. La contrainte que l'on doit appliquer pour faire opérer une telle source de dislocations vaut:

$$
\tau=\frac{\mu \mathrm{b}}{\mathrm{D}}
$$

Cette dernière relation se généralise à de nombreuses situations dans lesquelles une dislocation peut contourner des obstacles distants de D. On peut ainsi modéliser dans une approche assez simpliste l'effet de différents types d'obstacles. Ainsi si les obstacles sont des précipités non cisaillables distants en moyenne de la distance L, leur franchissement s'effectue alors pas contournement, ce qui nécessite une contrainte : $\tau_{\text {cont }}=\frac{\mu \mathrm{b}}{\mathrm{L}}$. La distance L entre précipités est reliée simplement à leur fraction volumique $\mathrm{f}_{\mathrm{v}}$ et 
à leur rayon $\mathrm{R}: \tau_{\text {cont }}=\frac{\mu \mathrm{b}}{\mathrm{L}}=\mu \mathrm{b}\left(\frac{3}{2 \pi}\right)^{1 / 2} \sqrt{\mathrm{f}_{\mathrm{V}}} \frac{1}{\mathrm{R}}$. Pour les plus petits précipités (cohérents), ce mécanisme de contournement est en concurrence avec un franchissement par cisaillement qui requiert la contrainte $\tau_{\text {cis }}=\left(\frac{3 \pi^{2}}{32}\right)^{1 / 2} \frac{\gamma^{3 / 2}}{\mathrm{~b} \sqrt{T}} \sqrt{f_{\mathrm{V}}} \sqrt{\mathrm{R}}$, où $\gamma$ est l'énergie de surface du défaut créé dans le précipité cisaillé. La dépendance en $\mathrm{R}$ de ces deux relations permet de mettre en évidence l'existence d'un durcissement maximal, correspondant au pic de dureté des alliages durcis par précipitation [15].

De façon analogue, la relation (23) permet de mettre en évidence le rôle des dislocations pour l'écrouissage des métaux : c'est le durcissement par les dislocations de la forêt. Les dislocations présentes dans le cristal qui coupent le plan de glissement sont autant d'obstacles, ce sont les arbres de la forêt au travers desquels les dislocations du système actif doivent passer. La distance moyenne entre dislocations de la forêt s'exprime $\mathrm{d}_{\text {forêt }}=1 / \sqrt{\rho_{\text {forêt }}}$, on en déduit la contrainte nécessaire pour le franchissement de la forêt : $\sigma=\alpha \mu \mathrm{b} \sqrt{\rho_{\text {forêt }}}$, où $\alpha$ est un coefficient dont la valeur précise (autour de 0,3 ) dépend de la géométrie du glissement et du type d'interactions entre dislocations [16]. Cette relation connue sous le nom de loi de Taylor se généralise sous la forme :

$$
\sigma=\sigma_{0}+\alpha \mu \mathrm{b} \sqrt{\rho}
$$

et s'applique alors, de façon quasiment universelle, pour décrire l'écrouissage de très nombreux matériaux cristallins : métaux cubiques à faces centrés [17], cubiques centrés ou hexagonaux compacts [18] mais aussi composés covalents et ioniques [16] et composés intermétalliques [19].

\section{DÉFORMATION PLASTIQUE ET DISLOCATIONS}

La déformation plastique des matériaux cristallins résulte essentiellement du mouvement des dislocations. On distingue deux modes de mouvement : le premier, dit conservatif, n'implique pas de déplacement des atomes du cristal (leur mouvement reste inférieur à une distance interatomique) on parle alors de glissement. Ce mode de mouvement des dislocations est le plus facile à mettre ne œuvre, c'est donc le mode le plus courant à basse température. Les déformations créées sont exclusivement des déformations de cisaillement, ce qui implique que la déformation par glissement des dislocations se fait à volume constant. Ainsi, pour ce type de déplacement, les dislocations ne sont sensibles qu'à la partie déviatorique du tenseur des contraintes. Un second mode de mouvement des dislocations implique au contraire un transport de matière, grâce à la diffusion de lacunes ou d'interstitiels, il s'agit de la montée des dislocations. Ce mouvement de montée est qualifié de mouvement non conservatif car au cours de la montée des dislocations des lacunes (ou des interstitiels) sont consommées ou émises (émis ou consommés). On peut ainsi avoir des changements de volume du cristal et la montée est sensible à la pression hydrostatique locale, contrairement au glissement. Comme la diffusion est mise en jeu, il est clair que le mouvement par montée est plutôt actif à haute température (souvent dans des situations de fluage et/ou associées à de faibles vitesses de déformation).

\subsection{Glissement et système de glissement}

\subsubsection{Notion de système de glissement}

Le glissement des dislocations est un mouvement qui opère sans qu'aucun atome ne se déplace de plus d'une distance interatomique. Comme illustré sur la figure 7, la dislocation se déplace d'une position par le simple basculement d'une liaison interatomique. Un tel mouvement n'est possible que dans un plan qui contient le vecteur de Burgers de la dislocation (si d $\mathbf{S}$ est le vecteur normal à la surface balayé par la dislocation, $\mathrm{d} \mathbf{S} \cdot \mathbf{b}$ représente le volume de matière déplacé [1], on doit donc avoir ici $\mathrm{d} \mathbf{S} \cdot \mathbf{b}=0$ ). Le plan de glissement (PG) est donc le plan défini par le vecteur de ligne et le vecteur de Burgers de la dislocation. Ce plan est unique pour une dislocation non vis. Pour une dislocation vis, plusieurs plans de glissement sont possibles (formellement tout plan en zone avec b) tant que la dislocation n'est pas 


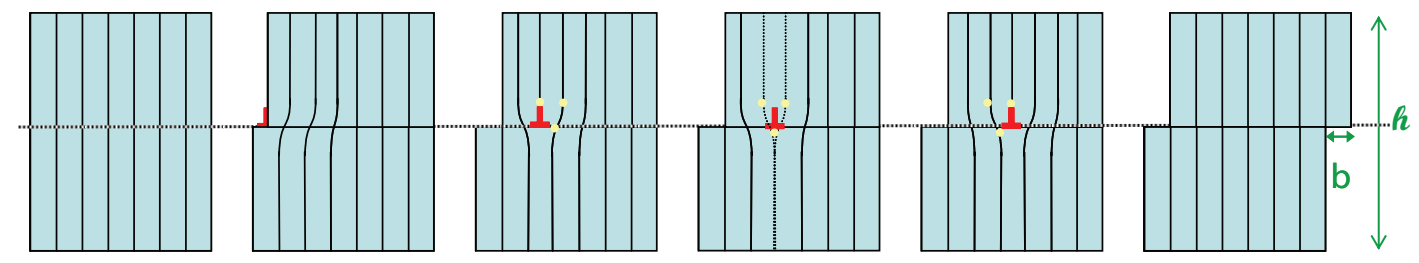

Figure 7. Schéma d'un monocristal cisaillé de part en part par le glissement d'une dislocation coin.

dissociée. Pour une dislocation dissociée le plan de glissement est défini par le plan de dissociation, une telle dislocation peut changer de plan de glissement en se recombinant (au moins localement) pour se redissocier dans un autre plan en zone avec b. Ce mécanisme que l'on appelle le «glissement dévié » est primordial pour la mise en place de structures tridimensionnelles de dislocations.

La géométrie du glissement impose un cisaillement qui a lieu dans une direction particulière, le vecteur de Burgers b, et selon un plan déterminé, le plan de glissement PG. Ce couple b, PG définit le «système de glissement». Comme les vecteurs de Burgers b doivent les plus courts possibles, leur direction correspond à des directions denses, et les plans de glissement des plans compacts, d'où il résulte que les systèmes de glissement susceptibles d'être activés sont imposés par la structure cristallographique. Ces systèmes sont rappelés dans le tableau 1 pour les trois principales structures métalliques. Ainsi dans les structures cubiques à faces centrées les systèmes de glissement sont du type $\langle 110\rangle\{111\}$. Pour pouvoir assurer une déformation générale, condition indispensable pour éviter les incompatibilités de déformation au sein d'un polycristal, il faut qu'au sein de chaque grain on puisse activer au moins cinq systèmes de glissement différents. Ces cinq systèmes correspondent aux cinq degrés de liberté d'une déformation plastique générale : six composantes du tenseur de déformation moins une car la conservation du volume implique une relation entre ces composantes. Cette règle connue sous de nom de «principe de Von Mises» fournit une première explication à la fragilité des métaux hexagonaux pour lesquels seul le glissement basal (3 systèmes) peut être activé à basse température.

\subsubsection{Loi d'Orowan}

Lorsque, comme sur la figure 7 un cristal de hauteur h est entièrement cisaillé par une dislocation de vecteur de burgers $\mathbf{b}$, il est immédiat d'établir le taux de déformation (cisaillement) qui en résulte : $\gamma=\mathrm{b} / \mathrm{h}$. Considérons à présent qu'une dislocation (i) ne traverse pas entièrement le cristal mais $\mathrm{y}$ parcourt une distance $\mathrm{X}_{\mathrm{i}}$ (figure 8(a)). La déformation est alors proportionnelle à la distance balayée $X_{i}: \gamma_{i}=\frac{b}{h} \frac{X_{i}}{\ell}$. Dans le cas de $N$ dislocations qui parcourent chacune une distance $X_{i}$ (figure 8(b)) on a

Tableau 1. Systèmes de glissement pour les trois principales structures métalliques (les systèmes indiqués en italiques ne sont pas activés à basse température).

\begin{tabular}{|c|c|c|c|}
\hline & Direction & Plan & $\begin{array}{c}\text { nombre de } \\
\text { systèmes }\end{array}$ \\
\hline CFC & $\langle 110\rangle$ & $\{111\}$ & 12 \\
\hline CC & $\langle 111\rangle$ & $\{110\}$ & 12 \\
& & $\{112\}$ & 12 \\
\hline & $\langle 11 \overline{2} 0\rangle=\mathbf{a}$ & $\{0001\}$ basal & 3 \\
& & $\{10 \overline{1} 0\}$ prismatique & 3 \\
HC & & $\{10 \overline{1} 1\}$ pyramidal I & 6 \\
& $\langle 11 \overline{2} 3\rangle=\boldsymbol{a}+\boldsymbol{c}$ & $\{11 \overline{2} 2\}$ pyramidal II & 6 \\
\hline
\end{tabular}




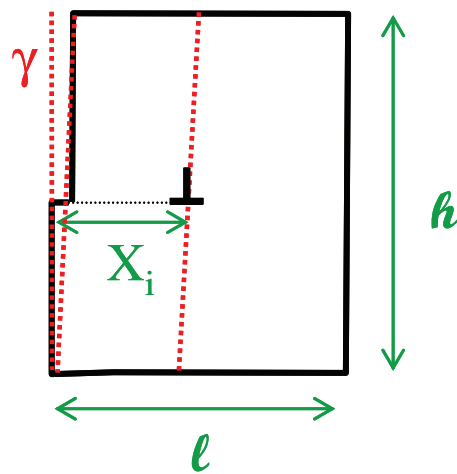

(a)

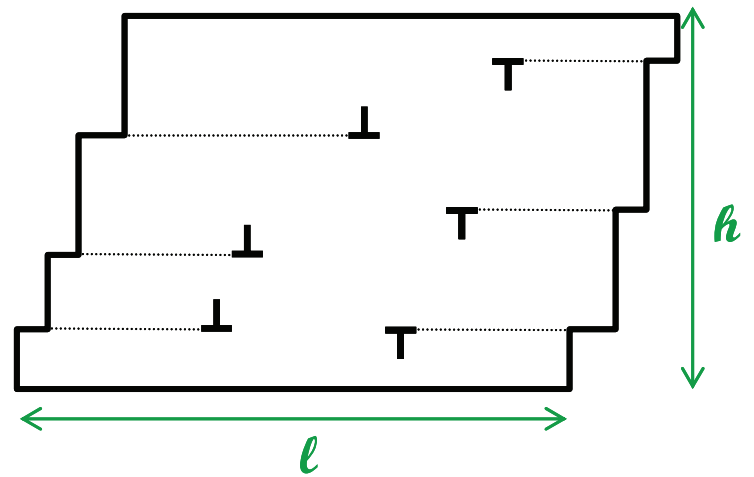

(b)

Figure 8. Géométrie d'un cristal cisaillé partiellement par une seule dislocation (a) ou par plusieurs dislocations (b).

donc :

$$
\gamma=\sum_{\mathrm{i}} \gamma_{\mathrm{i}}=\sum_{\mathrm{i}} \frac{\mathrm{b}}{\mathrm{h}} \frac{\mathrm{X}_{\mathrm{i}}}{\ell}=\frac{\mathrm{b}}{\mathrm{h}} \frac{\mathrm{N}\langle\mathrm{X}\rangle}{\ell}=\frac{\mathrm{NP}}{\mathrm{P}} \frac{\mathrm{b}}{\mathrm{h}} \frac{\langle\mathrm{X}\rangle}{\ell}=\rho \mathrm{b}\langle\mathrm{X}\rangle
$$

où $\mathrm{P}$ est la profondeur du cristal et $\langle\mathrm{X}\rangle$ la distance moyenne parcourue par chaque dislocation au sein du cristal. Cette relation est plus connue sous sa forme dérivée temporelle sous le nom de loi d'Orowan :

$$
\dot{\gamma}_{\mathrm{p}}=\rho_{\mathrm{m}} \mathrm{bv}
$$

Cette loi est d'une importance primordiale en plasticité puisqu'elle relie directement la grandeur macroscopique qu'est la vitesse de déformation plastique $\dot{\gamma}_{\mathrm{p}}$ avec des paramètres internes à la microstructure que sont la densité de dislocation mobiles $\rho_{\mathrm{m}}$ et leur vitesse moyenne v. Nous noterons qu'une déformation macroscopique est associée à un mouvement quelconque d'une dislocation au sein du cristal, sans que celle-ci ne débouche nécessairement sur une surface. Tout mouvement de dislocation correspond à une déformation, la grandeur pertinente est en fait l'aire balayée par la ligne de dislocation au cours de son déplacement.

\subsubsection{Contrainte et déformation résolue. Facteur de Schmid}

La déformation macroscopique $\gamma$ qui intervient dans la loi d'Orowan, de même que la contrainte de cisaillement responsable de la composante de glissement de la force de Peach-Kölher ressentie par une dislocation ( $\$ 3.2 \mathrm{ci}$ - dessus), sont exprimée en projection dans le plan de glissement et selon le vecteur de Burgers de la dislocation. On parle de contrainte et déformation projetées ou résolues (Resolved Shear Stress and Strain). Ces coordonnées sont liées à la contrainte $\sigma$ et la déformation $\varepsilon$ macroscopiques via les angles $\phi$ et $\lambda$ respectivement entre l'axe de chargement (représenté par la force F sur la figure 9) et la normale au plan de glissement d'une part et l'axe de chargement et la direction de glissement (le vecteur b) d'autre part. La projection géométrique s'écrit :

$$
\left\{\begin{array}{l}
\tau=\mathrm{F}_{\text {projeté }} / \mathrm{S}_{\text {projeté }}=\mathrm{F} \cdot \cos \lambda /(\mathrm{S} / \cos \phi)=\sigma \cdot \cos \lambda \cos \phi=\sigma \cdot \mathrm{m} \\
\gamma=\Delta \ell_{\text {projeté }} / \ell_{\text {projeté }}=\Delta \ell / \cos \lambda / \ell \cdot \cos \phi=\varepsilon /(\cos \lambda \cos \phi)=\varepsilon / \mathrm{m}
\end{array}\right.
$$

Où nous avons introduit le facteur : $m=\cos \lambda \cos \phi$, que l'on appelle le facteur de Schmid (on vérifie géométriquement que : $|\mathrm{m}| \leq 1 / 2)$.

Lorsque l'on sollicite en traction un monocristal selon une direction donnée, on peut ainsi calculer les facteurs de Schmid de tous les systèmes susceptibles d'être activés. Ce sont les systèmes possédant les facteurs de Schmid les plus élevés (en valeur absolue) qui seront activés en premier. La loi de Schmid 


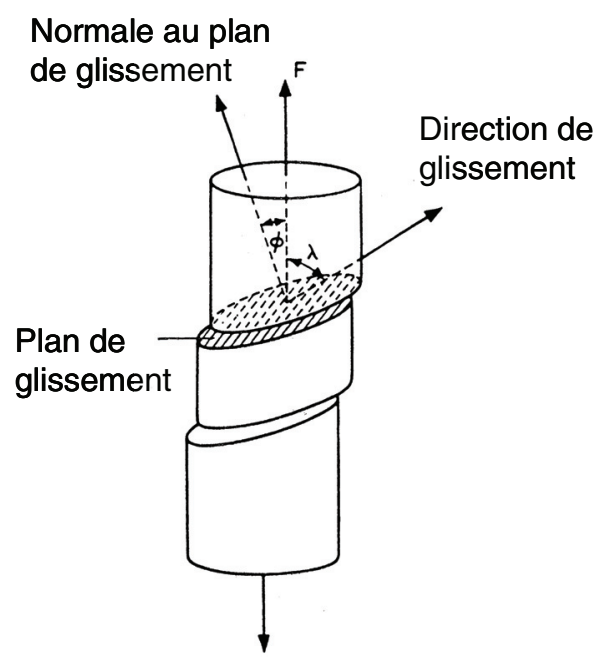

Figure 9. Géométrie d'un monocristal soumis à une sollicitation mécanique montrant la contrainte macroscopique selon le système de glissement (les trois directions: axe de sollicitation, normale au plan de glissement et direction de glissement ne sont en général pas coplanaires).

et Boas stipule que le glissement selon un système donné commence lorsque la contrainte projetée pour ce système atteint une valeur critique (notée CRSS - Critical Resolved Shear Stress). Selon l'orientation de la contrainte macroscopique on peut avoir un système particulier qui possède un facteur de Schmid significativement plus élevé que tous les autres ; la déformation plastique aura alors lieu par activation de ce seul système de glissement, on parle de glissement simple (c'est le cas lors de la sollicitation d'un monocristal de structure cfc le long d'un axe $\langle 123\rangle$ ). Par opposition on parle de glissement multiple lorsque plusieurs systèmes sont activés simultanément. Notons que lors de la déformation plastique en glissement simple l'axe moyen du cristal a tendance à tourner pour se rapprocher de la direction de glissement. Ainsi, dans le cas des métaux cfc on passe de glissement simple à un glissement multiple, ce qui se traduit sur la courbe de traction par les stades I et II de durcissement [3]. Par extension, ce type de rotation du réseau peut donner naissance à la texture cristallographique des tôles laminées.

Dans le cas de la déformation plastique des polycristaux, on peut ramener la contrainte macroscopique $\sigma$ à une contrainte projetée moyenne $\langle\tau\rangle$ selon l'ensemble des systèmes de glissement possibles : $\sigma=\mathrm{M} \cdot\langle\tau\rangle$. Le facteur de proportionnalité $\mathrm{M}$, nommé facteur de Taylor [20] dépend alors des systèmes possibles donc du type de réseau cristallin pour un polycristal isotrope $(M=3,06$ pour un cfc - [3]) et de l'orientation moyenne des grains donc de la texture éventuelle (e.g. pour le cas des cfc on a les valeurs : $M>2,45$ et $M>3,67$ pour des textures de type (100) et (111) respectivement - voir la figure 33 dans [21]).

\subsection{Modélisation de la déformation plastique}

La loi d'Orowan décrite par l'équation (26) relie la vitesse de déformation plastique à la densité de dislocations mobiles et à la vitesse moyenne des dislocations. Ces deux paramètres (vitesse et densité) qui caractérisent les dislocations au sein du matériau gouvernent donc la déformation plastique, c'està-dire le comportement macroscopique. La modélisation de la déformation plastique des matériaux par des mécanismes de mouvement des dislocations nécessite donc de prendre en compte à la fois ces deux aspects. Ce type de modélisation est assez complexe et nécessite en général des outils numériques de simulation tels que les codes de dynamique des dislocations (voir l'article de G. Monnet dans cet 


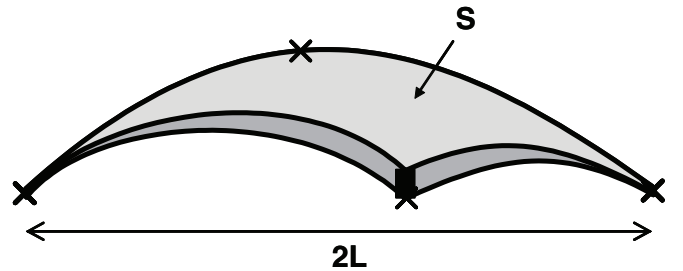

(a)

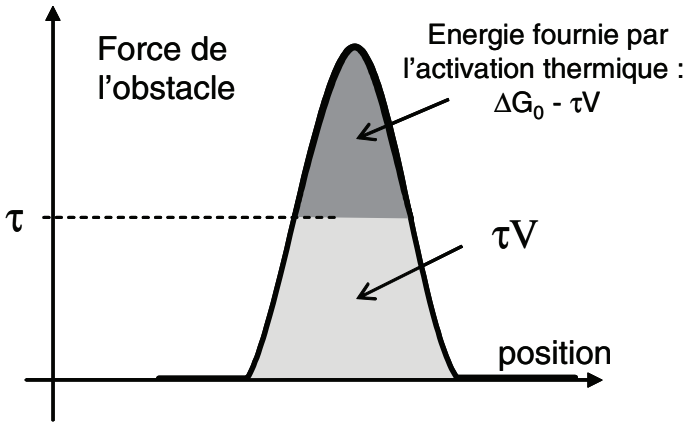

(b)

Figure 10. Schémas du franchissement des obstacles localisés selon la modélisation de l'activation thermique. En (a) la dislocation franchit l'obstacle central : la surface hachurée correspond à la surface d'activation alors que la surface $\mathrm{S}$ (surface grisée + surface hachurée) correspond à l'aire balayée. Le schéma d'énergie présenté en (b) montre comment l'énergie totale de franchissement se répartit entre le travail de la contrainte $\tau \mathrm{V}$ (zone grisée) et l'activation thermique (zone hachurée).

ouvrage). Dans le cadre de cet article où nous souhaitons montrer les différents outils utilisés pour la modélisation, nous allons examiner séparément les effets de vitesse et de densité. En effet ces deux approches ont longtemps été utilisées et sont souvent justifiées car, dans de nombreuses situations, le comportement mécanique est dicté soit par la mobilité individuelle (éventuellement moyennée) des dislocations - on est alors dans le cadre de modèles en vitesse - ou par le comportement collectif d'un ensemble de dislocations - modèles en densité.

\subsubsection{Vitesse des dislocations et activation thermique}

La vitesse des dislocations qui apparaît dans la loi d'Orawan est une vitesse moyenne de déplacement. Au sein du cristal, le mouvement des dislocations est gêné par la présence d'obstacles localisés et la mobilité des dislocations dépend essentiellement de la fréquence de franchissement de ces obstacles. Le formalisme de l'activation thermique que nous introduisons ici permet de modéliser le franchissement en prenant en compte les effets combinés de la contrainte et de la température. Nous ne donnons ici que quelques grandes lignes de ce formalisme, une description bien plus complète et des exemples d'applications à différents mécanismes de franchissement et différents types d'obstacle sont donnés dans un récent ouvrage spécifiquement dédié à l'activation thermique dans la plasticité des cristaux [22].

La figure 10 illustre la situation d'une dislocation épinglée par des obstacles et qui franchit l'obstacle central, ce qui lui permet de balayer la surface $\mathrm{S}$. La vitesse moyenne de la dislocation correspond donc à la surface $\mathrm{S}$ parcourue divisée par la longueur moyenne de dislocations entre deux obstacles $\mathrm{L}$ et le temps nécessaire pour le déplacement. Ce temps est la somme d'un temps d'attente $\mathrm{t}_{\mathrm{a}}$ (temps nécessaire pour franchir un obstacle) et le temps de vol $\mathrm{t}_{\mathrm{vol}}$ (temps mis par la dislocation pour parcourir librement la distance entre deux obstacles). Le temps de vol étant négligeable devant le temps d'attente, la vitesse moyenne des dislocations est donc directement reliée à la fréquence $v$ de franchissement des obstacles :

$$
\mathrm{v}=\frac{\mathrm{S}}{\mathrm{L}} \frac{1}{\left(\mathrm{t}_{\mathrm{a}}+\mathrm{t}_{\mathrm{vol}}\right)} \approx \frac{\mathrm{S}}{\mathrm{L}} \frac{1}{\mathrm{t}_{\mathrm{a}}} \approx \frac{\mathrm{S}}{\mathrm{L}} v
$$

Dans le cadre de la théorie de l'activation thermique, les obstacles sont représentés par une barrière énergétique, caractérisée par une énergie libre de Gibbs $\Delta \mathrm{G}$. La fréquence de franchissement s'écrit 
donc comme le produit de la fréquence d'attaque par la probabilité de franchissement : $v=v_{\text {attaque }} \mathrm{P}=$ $\frac{\nu_{\mathrm{D}} \mathrm{b}}{\mathrm{L}} \exp \left[-\frac{\Delta \mathrm{G}}{\mathrm{kT}}\right]$, où $\nu_{\mathrm{D}}$ est la fréquence de Debye. Ainsi la loi d'Orowan peut se réécrire :

$$
\dot{\gamma}=\mathrm{A} \rho_{\mathrm{m}} \mathrm{b} \frac{\mathrm{S}}{\mathrm{L}} \frac{\nu_{\mathrm{D}} \mathrm{b}}{\mathrm{L}} \exp \left[-\frac{\Delta \mathrm{G}}{\mathrm{kT}}\right]=\dot{\gamma}_{0} \exp \left[-\frac{\Delta \mathrm{G}}{\mathrm{kT}}\right]
$$

Dans ce traitement, il faut tenir compte du fait que la contrainte $\tau$, que ressent la dislocation, participe au franchissement des obstacles. On introduit donc la hauteur énergétique de l'obstacle sous contrainte nulle $\left(\Delta \mathrm{G}_{0}\right)$ et le volume d'activation $(\mathrm{V})$.

$$
\Delta \mathrm{G}=\Delta \mathrm{G}_{0}-\tau \mathrm{V} .
$$

Le volume d'activation s'exprime souvent en unité de $b^{3}$, la valeur ainsi obtenue permet de se faire une idée du nombre d'atomes impliqués collectivement dans le processus de franchissement. On exprime aussi cette grandeur comme une aire d'activation $(\mathrm{V} / \mathrm{b})$ qui co rrespond à l'aire balayée par la dislocation au cours du franchissement (c'est l'aire hachurée sur la fig. 10(a)). Le volume d'activation peut être mesuré expérimentalement au cours d'un essai de déformation macroscopique en imposant des transitoires de déformation. Au cours d'un essai à vitesse imposée, un saut de vitesse ou un essai de relaxation de contrainte simple permettent d'obtenir un volume d'activation « apparent », alors qu'un essai de relaxations et/ou de fluages répétés permet d'obtenir un volume «effectif» pour lequel on tient compte des variations éventuelles de microstructure [23]. Les valeurs de volumes d'activation ainsi mesurées, et surtout les variations de ce volume en fonction de la température et/ou de la contrainte, permettent d'avoir des indications sur les mécanismes microscopiques qui font obstacle au mouvement des dislocations et sur les changements éventuels de mécanismes (par exemple selon les régimes de température, comme dans le cas de la phase $\gamma$ - TiAl [24]). Comme l'indique l'équation (30) une valeur faible de $\mathrm{V}$ correspond à une forte activation thermique, c'est-à-dire un mécanisme fortement influencé par la température. Ainsi des mouvements de dislocations contrôlés par des mécanismes de montée sont caractérisés par des volumes de l'ordre de l'unité alors que si la déformation est gouvernée par des interactions entre dislocations sécantes (durcissement de forêt) les volumes d'activation associés peuvent prendre des valeurs de plusieurs milliers de $b^{3}$.

Le volume d'activation qui apparaît dans l'expression (30) se définit plus formellement comme un coefficient thermodynamique accessible expérimentalement :

$$
\mathrm{V}=\frac{\partial(\Delta \mathrm{G})}{\partial \sigma}=\mathrm{kT} \frac{\partial(\ln \dot{\varepsilon})}{\partial \sigma}
$$

Cette expression peut être rapprochée de l'écriture du coefficient de sensibilité à la vitesse de déformation : $\mathrm{S}=\partial(\ln \sigma) / \partial(\ln \dot{\varepsilon})$. Ces deux coefficients sont bien sûr liés (on a : $\mathrm{S}=\mathrm{kT} / \sigma \mathrm{V}$ ) et reflètent tous deux le lien entre la contrainte d'écoulement et la vitesse de déformation, donc le temps, signifiant ainsi le caractère visqueux de la déformation plastique. S'il est présent pour la plupart des mécanismes de déformation plastique par mouvement de dislocations, il est évident que ce caractère visqueux acquiert une importance prépondérante lorsque la température augmente et sous faible charge - c'est le cas de sollicitations en fluage. Ainsi dans le cadre de la théorie de l'activation thermique du mouvement des dislocations, il y a stricte continuité de mécanisme entre fluage et essais à vitesse de déformation imposée.

\subsection{2 Évolution de la densité de dislocations}

Rappelons encore que c'est le déplacement des dislocations au sein des cristaux qui induit leur déformation plastique. La distance parcourue (ou plus précisément l'aire balayée) au sein d'un cristal ou d'un grain par une dislocation étant nécessairement finie, il faut pour continuer à déformer plastiquement un matériau cristallin multiplier les dislocations. Un processus de déformation plastique peut donc être décrit comme la création de nouvelles dislocations suivie de leur déplacement. A l'issue de ce 
déplacement ces dislocations peuvent être stockées dans le matériau, déboucher à la surface libre en créant une marche ou s'annihiler avec des dislocations déjà présentes dans le cristal. Nous avons vu que la contrainte d'écoulement est directement liée à la densité totale de dislocations présentes au sein du cristal via la relation de Taylor (équation (24)). Ainsi stocker des dislocations dans le cristal correspond à un écrouissage, alors que l'annihilation de dislocations correspond à un adoucissement du matériau. Kocks et Mecking ont ainsi étudié les différents aspects de l'écrouissage des métaux de structure cubique à faces centrées en terme d'évolution des densités de dislocations [21]. La densité totale de dislocation $\rho_{t}$ peut être scindée en deux populations distinctes : les dislocations mobiles $\rho_{\mathrm{m}}$ et les dislocations immobiles $\rho_{\text {im }}$. Schématiquement on peut dire que les dislocations mobiles dont la densité apparaît dans la loi d'Orowan pilotent la vitesse de déformation plastique, alors que les dislocations immobiles interviennent sur la contrainte d'écoulement en créant une contrainte à longue distance au sein du matériau (contrainte interne). L'évolution de ces deux populations de dislocations est dictée par quatre mécanismes différents : la multiplication qui crée des dislocations mobiles $\left(\dot{\rho}_{\text {mult }}\right)$, l'épuisement lorsqu'une dislocation mobile est bloquée dans le cristal ( $\left.\dot{\rho}_{\text {èpuis }}\right)$, l'annihilation d'une dislocation mobile avec une autre dislocation - supposée immobile - $\left(\dot{\rho}_{\text {annih }}\right)$ et enfin les dislocations émergeant à une surface libre du cristal $\left(\dot{\rho}_{\text {sortie }}\right)$. Ce qui donne une évolution globale des deux populations :

$$
\left\{\begin{array}{l}
\dot{\rho}_{\mathrm{m}}=\dot{\rho}_{\text {mult }}-\dot{\rho}_{\text {èpuis }}-\dot{\rho}_{\text {annih }}-\dot{\rho}_{\text {sortie }} \\
\dot{\rho}_{\text {im }}=\dot{\rho}_{\text {èpuis }}-\dot{\rho}_{\text {annih }}
\end{array}\right.
$$

La modélisation du comportement mécanique consiste alors à écrire ces lois d'évolution, puis à résoudre l'équation d'Orowan, en prenant en compte les conditions expérimentales (essai à vitesse de déformation imposée, fluage ....). Souvent on peut négliger les termes d'annihilation et de sortie (en particulier si l'on s'intéresse au début de la déformation plastique). De nombreux exemples sont disponibles dans la littérature où ce type d'approche a été utilisé :

- pour expliquer l'anomalie de limite d'élasticité par épuisement de dislocations mobiles dans des composés intermétalliques ( $\gamma$ - TiAl [25], $\left.\mathrm{Ni}_{3} \mathrm{Al}[26]\right)$.

- trouver les lois pertinentes de multiplication des dislocations dans le germanium [27].

- modéliser les courbes de fluage et de traction d'un alliage industriel (Udimet 720) et comprendre les effets d'une variation microstructurale sur le comportement des dislocations et la réponse mécanique du matériau [28].

\subsection{Montée, crans et lacunes}

Nous avons examiné jusqu'à présent le mouvement conservatif des dislocations, c'est-à-dire le glissement. Ce glissement est géométriquement restreint dans le plan de glissement. Alors qu'une dislocation vis peut changer de plan de glissement par glissement dévié, les dislocations coins (et par extension toute dislocation avec une composante coin) ont un plan de glissement unique. Un mouvement hors du plan de glissement nécessite le transport d'atomes à longue distance puisqu'un plan atomique s'étend ou est consommé.

La montée pure correspond ainsi à un mouvement le long de ce plan consommé (ou qui s'étend). On qualifie de montée positive un mouvement qui consomme le plan atomique (c'est le cas de la montée illustrée sur la figure 11(a)) ; une montée positive s'accompagne donc d'une consommation de lacunes (ou de création d'interstitiels). Cette montée peut être provoquée mécaniquement, en effet la force de Peach - Kölher créée par une contrainte normale parallèle au vecteur de Burgers (fig. 11(a)) est une force perpendiculaire au plan de glissement PG, c'est une force de montée $\left(\mathbf{F}_{\mathbf{M}}\right)$.

Une dislocation animée d'un mouvement de montée positive consomme les lacunes environnantes, ce qui modifie la concentration en lacune c qui s'écarte de sa valeur à l'équilibre $\mathrm{c}_{0}$. Il est évident que si la concentration locale de lacunes décroît il sera d'autant plus difficile de faire monter la dislocation. Alternativement si on place une dislocation dans un cristal sursaturé en lacunes, celle-ci aura tendance à monter pour absorber ces lacunes et en ramener la concentration à sa valeur d'équilibre. Ces interactions 


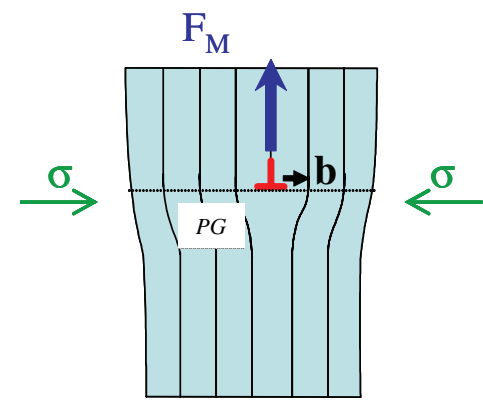

(a)

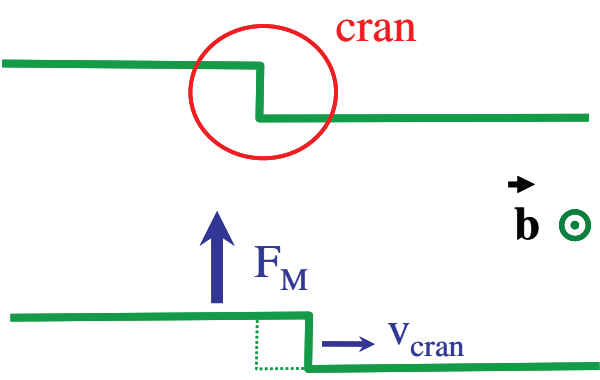

(b)

Figure 11. La montée d'une dislocation coin peut être provoquée par une contrainte de compression (a) qui engendre une force $\mathbf{F}_{\mathbf{M}}$ perpendiculaire au plan de glissement. La montée de la ligne de la dislocation ne s'effectue pas de façon rigide mais par mouvement de crans (b).

se traduisent par une «force chimique » ou «osmotique» de montée qui s'exprime [1,22] :

$$
\mathrm{F}_{\mathrm{O}}=\frac{\mathrm{kTb}}{\Omega} \ln \frac{\mathrm{c}}{\mathrm{c}_{0}},
$$

$\mathrm{F}_{\mathrm{O}}$ est une force par unité de longueur de ligne de la dislocation, $\Omega$ représente le volume atomique $\left(\Omega=\mathrm{b}^{3}\right)$.

Il apparaît évident que la ligne de dislocation ne peut pas monter de façon rigide sur toute sa longueur (ce qui nécessiterait l'absorption simultanée d'un très grand nombre de lacunes le long de la ligne). La montée se fait en réalité par le mouvement de singularités le long de la ligne de dislocation, que l'on appelle des crans (jogs en anglais). Ces crans sont des segments de ligne de dislocation hors du plan de glissement, un cran élémentaire a une hauteur $(\mathrm{h})$ égale à une distance interatomique $(\mathrm{h}=\mathrm{d} \approx \mathrm{b})$. La figure 11(b) montre un tel cran sur une dislocation coin et comment une force de montée implique le mouvement du cran.

Les crans peuvent être créés lors de la déformation plastique des cristaux lorsqu'une dislocation mobile coupe des dislocations de la forêt. Dans certaines configurations, ces crans ne peuvent pas accompagner le mouvement de glissement de la ligne de dislocation, par exemple les crans sur la dislocation de la figure 12 se déplacent par montée en émettant des lacunes. Pour une faible concentration de crans (i. e. pour une distance entre crans $\lambda$ grande devant la distance moyenne entre dislocations), la force de glissement qui s'exerce sur le segment $\lambda$ germet de générer les lacunes nécessaires au mouvement du cran (le travail mécanique de la force de Peach et Kölher lorsque la dislocation avance d'une distance $b$ est supérieur à l'enthalpie de formation des lacunes $-\sigma_{\mathrm{ap}} \mathrm{b}^{2} \lambda>\mathrm{H}_{\mathrm{F}}$ ). Ainsi la déformation plastique «à froid» peut produire une forte densité de défauts ponctuels au sein du matériau.

Lors de la sollicitation mécaniques à haute température et faible contrainte, la densité de crans peut devenir beaucoup plus importante, la diffusion des défauts ponctuels est alors nécessaire et devient le mécanisme qui contrôle la vitesse de déplacement des dislocations. Barret et Nix [29] ont ainsi proposé une modélisation du fluage à partir du mouvement de dislocations vis saturées en crans. Ce modèle a été plus récemment repris et complété pour s'appliquer à d'autres matériaux ( TiAl [30], Ti [31] et Zr [32]) en prenant en compte des crans de hauteurs variables. Enfin, dans certaines situations, la déformation plastique est principalement contrôlée par la montée pure de dislocations. C'est le cas par exemple lors de la déformation à vitesse imposée de composés quasicristallins [33] ainsi que pour le fluage de superalliages à hautes températures où les précipités $\gamma$ ' sont déformés par la montée de dislocations comme illustré sur la figure 13. 


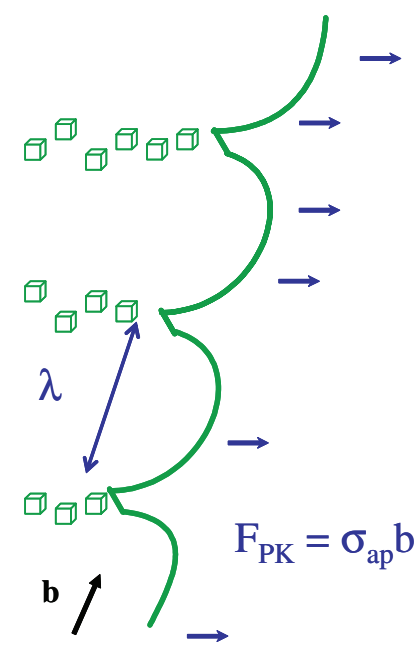

Figure 12. Schéma d'une dislocation comportant des crans ; les segments courbes se déplacent par glissement et traînent les crans qui créent des défauts ponctuels.

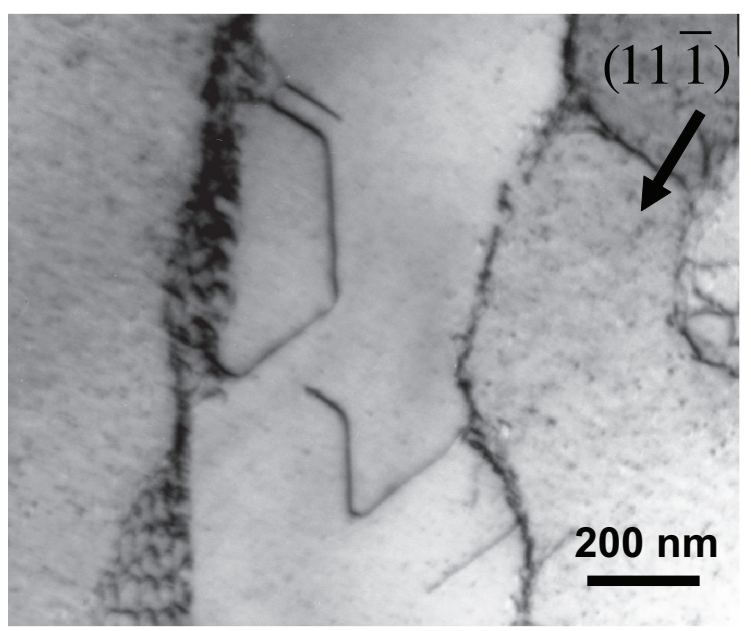

Figure 13. Dislocations de type $\mathbf{b}=\mathrm{a}[010]$ qui traversent par montée un précipité $\gamma$ ' d'un superalliage MC2 sollicité en fluage le long de l'axe [001] à $1150^{\circ} \mathrm{C}$ [34].

\section{CONCLUSIONS}

Nous avons dans cet article donné les bases de la théorie des dislocations qui permet de décrire et d'essayer de comprendre à l'échelle mésoscopique le comportement mécanique macroscopique des métaux. Nous montrons ainsi que des calculs relativement simples permettent d'approcher des comportements macroscopiques de différents degrés de complexité :

- notion de contrainte seuil (comportement mécanique de type idéalement plastique) pour le durcissement par précipitation ;

- notion de durcissement par les lois d'évolution de la densité de dislocations (à rapprocher des modèles plastiques avec écrouissage des mécaniciens) ; 
- prise en compte de la viscosité via le formalisme de l'activation thermique, définition des grandeurs thermodynamiques - volumes et énergie d'activation.

Ces différents mécanismes, et d'autres basés sur le comportement des dislocations seront repris et explicités pour présenter le rôle des dislocations et leur implication dans les phénomènes d'oxydation et de corrosion présentés dans les différents articles de cet ouvrage.

\section{Références}

[1] Friedel J. and Dislocations (Pergamon Press, Oxford, 1964).

[2] Hirth J.P. and Lothe J., Theory of Dislocations (Wiley, NewYork, 1982).

[3] Jaoul, B. Etude de la plasticité et application aux métaux (Paris Tech Les Presses, Paris 2008).

[4] Dieter G.E., Mechanical Metallurgy (Mc Graw Hill, New York, 1986).

[5] Douin J., Mécanique des milieux continus, Introduction à la plasticité des matériaux (Diderot, Paris 1997).

[6] Martin J.L., Dislocations et plasiticité des cristaux (PPUR Lausanne, 2000).

[7] Jouffrey B., «Historique de la notion de dislocation» Dislocations et déformation plastique, Yravals 1979, Groh, Kubin, Martin Eds (Ed. de Physique, Paris, 1980) pp. 1-16.

[8] Kléman M., Points Lignes Parois (Ed. de Physique, Paris, 1977).

[9] Douin J., programme Disdi : http://www.cemes.fr/Personnel/douin/Disdi-Page.html

[10] Interfaces and Plasiticity, Clément et Douin éditeurs (Scitec Publications, Zürich, 1998).

[11] Beauchamp, Pet Lépinoux, J., Phil. Mag. A 77 (1998) 541.

[12] Lépinoux J. in ref 10 (1998) pp. 115-132.

[13] Monnet G., cet ouvrage.

[14] Pettinari F., Couret A., Caillard D., Clément N. et Coujour A., J. Microscopy, 203 (2001) 47.

[15] Strudel J.L., «Mechanical Properties of Multiphase Alloys » in Physical Metallurgy, Caan et Haasen Eds (Elsevier, Amsterdam, 1996), pp. 2105.

[16] Lavrentev F.F., Mater. Sci. Eng. A 46 (1980) 191.

[17] Mecking H. et Kocks U.F., Acta Metall. 29 (1981) 1865.

[18] Orlova A., Acta Metall. Mater. 39 (1991) 2805.

[19] Viguier B., Mater. Sci. Eng. A 349 (2003) 132.

[20] Taylor G.I., J. Inst. Metals 62 (1938) 307.

[21] Kocks U.F. and Mecking H., Progress in Materials Science 48 (2003) 171.

[22] Caillard D. et Martin J.L., Thermally activated mechanisms in crystal plasticity (Pergamon Elsevier, Oxford 2003).

[23] Spätig P., Bonneville J. et Martin J.-L., Mater. Sci. Eng. A 167 (1993) 73.

[24] Viguier B., Bonneville J. et Martin J.L., Acta Mater. 44 (1996) 4403.

[25] Louchet F. et Viguier B., Phil. Mag. A 71 (1995) 1313.

[26] Louchet F., Mater. Sci. Eng. A 234 (1997) 275.

[27] Fikar J., Viguier B., Kruml T., et Dupas C., J. Physics - Cond. Matter 14 (2002) 12887.

[28] Terzi S., Couturier R., Guétaz L. and Viguier B., Mater. Sci. Eng. A 483-484 (2008) 598.

[29] Barret C.R. et Nix W. D., Acta Metall. 13 (1965) 1247.

[30] Karthikeyan S., Viswanathan G.B. and Mills M.J., Acta Mater. 52 (2004) 2577.

[31] Viswanathan G.B., Hayes R.W. and Mills M.J., Mater. Sci. Eng. A 319 (2001) 706.

[32] Moon J.H., Cantonwine P.E., Anderson K.R., Karthikeyan S. and Mills M.J., J. Nucl. Mater. 353 (2006) 177.

[33] Monpiou F. et Caillard D., Phil. Mag. 84 (2004) 2777.

[34] Touratier F., Thèse de l'Université de Toulouse, Institut National Polytechnique, Toulouse, 2009. 
